\title{
Intelligence collective et organisation co-dépendante : le rôle de l'expert-comptable dans le crowdlending
}

Héloïse Berkowitz (CNRS Toulouse School of Management)

Antoine Souchaud (NEOMA Business School)

\author{
Pre-Print : A paraitre dans CCA
}

\section{Citer comme :}

Berkowitz H., Souchaud A. 2019, Intelligence collective et organisation co-dépendante : le rôle de l'expert-comptable dans le crowdlending, Comptabilité Contrôle Audit, 25 (3) : 41-67.

\section{Résumé :}

Quel rôle 1'expert-comptable peut-il jouer dans l'utilisation de l'intelligence collective (IC) dans le crowdlending et à quelles conditions ? Cet article s'appuie sur une étude de cas approfondie d'une tentative inaboutie d'exploiter l'IC grâce à l'expertise-comptable dans le cadre d'un partenariat entre une plateforme de crowdlending et l'Ordre des Experts Comptables. Nos résultats décrivent certaines actions de l'EC permettant d'activer l'intelligence collective sur les forums, mais révèlent aussi deux facteurs organisationnels d'échec de l'exploitation de cette ressource, le non-respect des relations de co-dépendance et l'hypocrisie organisationnelle. Cette analyse nous conduit à proposer un modèle élargi de co-dépendance entre plateforme, porteurs de projet, foule et experts-comptables dans le crowdlending afin d'exprimer et d'exploiter l'IC.

Mots-clés : expert-comptable, intelligence collective, crowdlending, organisation partielle, organisation co-dépendante

\section{Title: Chartered-accountants and collective intelligence in the co-dependent} organization of crowdlending

\begin{abstract}
:
How can chartered accountants help use collective intelligence in crowdlending? This article studies a failed attempt to exploit collective intelligence thanks to chartered accountants in a partnership between a platform and the professionals' association. We identify chartered accountants' actions that can activate collective intelligence on
\end{abstract}


forums. We also reveal the organizational factors that explain the failure to exploit this resource, co-dependence relations non-respect and organizational hypocrisy. Based on this analysis, we propose a revisited model of crowdlending as a co-dependent organization between platform, entrepreneurs, crowd and chartered accountants allowing to enact and exploit collective intelligence.

Keywords: chartered accountant, collective intelligence, peer-to-peer lending, partial organization, co-dependent organization 


\section{Introduction}

«La foule peut-elle prendre des décisions intelligentes ? » s'interrogent Bertrand et Jakubowski (2016) dans leur étude du secteur du financement participatif. L'émergence du crowdlending, i.e. le financement participatif sous forme de prêt rémunéré, repose sur la possibilité donnée à une foule de contributeurs de prêter, contre intérêts, de l'argent à des projets portés par des TPE ou des PME. Des plateformes en ligne de crowdlending mettent en relation directe contributeurs et porteurs de projet. La foule est ainsi au cœur du fonctionnement de ce type de financement, comme elle l'est plus largement au sein de l'économie digitale, par exemple avec TripAdvisor (Kremer, Mansour, \& Perry, 2014). La foule interagit, évalue et note les acteurs de cette «économie collaborative ». Dans cette relation numérique, la foule peut être trompée, ce que redoutent le régulateur et les associations de consommateurs. Mais elle peut aussi constituer une ressource à exploiter : c'est le principe de l'intelligence collective (IC).

Le financement participatif fait intervenir, en concurrence ou en complémentarité avec les experts, deux nouveaux types d'acteurs dans le financement d'un projet de création d'entreprise : la foule et la plateforme (Bessière \& Stéphany, 2014; Nielsen, 2018). Dans ce secteur, les plateformes s'appuient donc sur les décisions d'investissement de la foule, c'est-à-dire sur l'IC. C'est, selon Nielsen (2018), parce que plateforme, porteurs de projet et foule sont partiellement organisés en une « organisation co-dépendante » que le financement participatif peut réussir. En effet, les interactions et le pouvoir de décision partagé sur l'appartenance à l'organisation codépendante, sa hiérarchie, ses règles, la surveillance de ses membres et leurs sanctions assurent un équilibre réussi à l'échelle du financement. Or ce modèle d'organisation codépendante tel que pensé par Nielsen semble d'emblée exclure l'expert-comptable (EC).

Pourtant en France, certaines plateformes ont essayé de s'appuyer sur 1'EC (Calme, Onnee, \& Zoukoua, 2018), connaisseur de données fines sur les PME, c'est-àdire sur les porteurs des projets à financer (Chapellier, 2003). Dans sa formulation actuelle, le modèle du crowdlending comme co-dépendance entre plateforme, porteurs de projet et foule ne rend pas compte de la place de l'EC, ni de son articulation potentielle avec l'IC. Notre question de recherche se formule donc en ces termes : quel rôle 1'EC peut-il jouer dans l'exploitation de l'IC dans le crowdlending et à quelles conditions?

La compréhension de la place de l'EC et de l'IC dans le crowdlending est largement naissante dans la littérature, ce secteur étant lui-même émergent et les 
données empiriques faisant encore défaut. Or les enjeux sont importants puisque cette activité a récemment remis en cause tout un monopole bancaire vieux de plus d'un demi-siècle, ouvrant ainsi la voie aux contributeurs individuels (Souchaud, 2017). En outre, le métier d'EC doit sans cesse s'adapter à un environnement en évolution constante et rapide (Cormier \& Magnan, 2005; Frey \& Osborne, 2017; Susskind \& Susskind, 2015). Dans cet article, nous étudions le rôle de l'EC dans l'expression et l'exploitation de l'IC. Notre objectif ultime est d'enrichir le modèle d'organisation codépendante de Nielsen (2018) en explorant la place possible pour la figure de l'EC.

Pour ce faire, nous avons mené une étude de cas approfondie dans le secteur du crowdlending français : l'échec du partenariat entre Lendopolis et l'Ordre des ExpertsComptables. Nous avons collecté un riche matériau sur plus de trois années, combinant entretiens avec les acteurs et collecte des échanges entre foule et porteurs de projets sur les forums de la plateforme. Nos résultats révèlent l'importance de l'EC dans l'expression de l'IC ainsi que les déviations de Lendopolis par rapport au modèle de Nielsen, déviations qui produisent de l'hypocrisie organisationnelle. Introduisant un déséquilibre dans les relations entre acteurs, Lendopolis perturbe la co-dépendance entre les acteurs du crowdlending. Sur la base de cette analyse, nous proposons un modèle élargi de co-dépendance entre plateforme, porteurs de projet, foule et EC permettant d'exploiter l'IC.

Notre étude contribue à la littérature à plusieurs titres. La mise en évidence de deux facteurs d'échec du partenariat, le non-respect de la co-dépendance et une forme d'hypocrisie organisationnelle, vient compléter l'analyse récente des logiques partenariales dans le crowdlending de Calme, Onnee et Zoukoua (2018). Nous contribuons également à la théorie des organisations en élargissant le modèle de Nielsen (2018) pour inclure la figure de l'EC. Plus généralement, notre utilisation des concepts d'organisation partielle et de co-dépendance vient enrichir les travaux passés sur le crowdfunding mobilisant la théorie de l'agence, des plateformes ou de l'IC. Nos travaux possèdent également des implications pratiques, ouvrant notamment des pistes pour un renouvellement de la profession d'EC dans le contexte de l'économie collaborative.

Dans la section suivante, nous proposons une revue de la littérature sur les rapports entre foule et expertise, en particulier dans le financement participatif présenté comme une organisation dite «co-dépendante ». Après avoir décrit la méthodologie de cette étude de cas, nous présentons les résultats qui sont ensuite discutés, à la lumière du modèle d'organisation co-dépendante. 


\section{Revue de la littérature}

Le développement des plateformes participatives repose sur un principe d'émancipation de la foule et un affaiblissement potentiel de l'expertise classique, c'està-dire professionnelle. Cette émancipation soulève des enjeux d'IC et de son expression en particulier dans le crowdlending, un sous-secteur émergent du financement participatif qui peut être pensé comme une organisation partielle faite de relations de codépendance.

\subsection{L'essor du «participatif » et l'articulation entre expertise et intelligence collective}

Grâce à la digitalisation et aux plateformes, l'essor du participatif conduit à des transformations profondes. La digitalisation, et plus spécifiquement la plateforme, constitue l'instrument principal de l'empowerment des foules (Dunleavy et al., 2006). Cette émancipation et cette mise à contribution de la foule trouvent leurs applications les plus étudiées dans le crowdsourcing, i.e. la production participative s'appuyant sur l'intelligence ou sur le savoir-faire d'un grand nombre de personnes (Chanal \& CaronFasan, 2010) ou encore sur l'innovation ouverte (Chesbrough, 2003).

La théorie classique de l'intelligence collective (IC) (Galton, 1907) montre que le groupe performe mieux que l'individu (pour prédire des évènements de marché, des scénarios géopolitiques, ou pour crowdsourcer des idées). Becker, Brackbill et Centola (2017) révèlent plus précisément que la foule devient plus intelligente si les individus communiquent entre eux. D'autres travaux s'intéressent également à la précision de l'IC par rapport aux individus (Surowiecki, 2004) ou à la performance relative de l'agrégation de multiples estimations de plusieurs personnes par rapport à celle d'un seul individu (Dolder \& Assem, 2017).

De nombreux dispositifs s'appuyant sur les nouvelles technologies exploitent ce pouvoir de l'IC (Bonabeau, 2009). C'est le cas des réseaux ou des communautés en ligne comme Wikipedia, un management réussi d'un nombre massif de contributeurs (Bonabeau, 2009) ou de Google qui s'appuie sur l'évaluation de millions d'utilisateurs pour produire des réponses intelligentes à leurs requêtes (Malone, Laubacher, \& Dellarocas, 2009). Dans cette perspective, une question centrale que soulève la littérature est celle de l'équilibre entre diversité et expertise et de la performance de l'une vis-à-vis de l'autre (Bonabeau, 2009; Malone et al., 2009). 


\subsection{La place de la foule et de l'expert dans le financement participatif}

Le financement participatif repose une hypothèse forte, celle de l'existence et de la possible mobilisation d'une IC (Bertrand \& Jakubowski, 2016). Cela pose des enjeux d'asymétrie d'information (Bessière \& Stéphany, 2014), par exemple si les porteurs de projet cachent des informations, ou fournissent des informations mensongères à la foule de contributeurs. Un des effets les plus significatifs est celui de sélection adverse, tel que formulé par Akerlof (1970). Plusieurs travaux récents explorent cette problématique, en étudiant par exemple les critères d'évaluation qu'utilise la foule pour investir dans des projets de crowdfunding (Mollick, 2013) ou le degré de divergence entre foule et experts (Mollick \& Nanda, 2015). D'après Mollick (2014), il n'est a priori pas acquis que les foules de contributeurs puissent prendre des décisions d'investissement à partir d'analyses de la qualité des projets. Mais son étude quantitative démontre que les contributeurs semblent réagir en majorité aux signaux de qualité des projets. Selon l'interprétation de l'auteur, c'est donc que les foules conditionnent bien leur décision de financement à une évaluation rationnelle des chances de réussite du projet, à l'instar des experts.

Kim et Viswanathan (2014) vont plus loin en arguant qu'une forme d'expertise réapparait au sein de la foule d'investisseurs dans le crowdfunding. Les auteurs nuancent la conception selon laquelle le financement participatif évacuerait totalement les mécanismes de décision basées sur l'expertise. Ils montrent au contraire que des formes d'expertise sont reconstruites au sein des foules, et que ces expertises influencent effectivement la prise de décision. En outre, Mollick et Nanda (2015) trouvent une concordance significative entre les décisions prises par les foules et par les experts, dans une étude du financement de projets de théâtre. Selon eux, lorsqu'il y a un désaccord, c'est souvent parce que la foule a décidé de financer un projet tandis que l'expert s'y refuse (Mollick \& Nanda, 2015).

S'intéressant aux processus de financement eux-mêmes, Bessière et Stéphany (2014) montrent que l'Equity Crowdfunding fonctionne par séquençage, avec à chaque étape du financement, une mobilisation des acteurs dans l'évaluation des projets. Les auteurs notent que si des divergences existent parmi les plateformes, la décision de financement résulte toujours des avis de la foule (Bessière \& Stéphany, 2014). Si les auteurs n'étudient pas les dynamiques d'évaluation de la foule, et la potentielle IC qui en résulte, ils rappellent que divers éléments peuvent diminuer le risque de sélection adverse : la syndication, les due diligences et le rôle de l'expertise. Relativement peu 
d'études explorent cependant les conditions organisationnelles de l'exploitation de l'IC dans le crowdlending.

\subsection{Le financement participatif comme organisation co-dépendante}

S'appuyant sur les travaux fondateurs d'Ahrne et Brunsson (2010), Nielsen (2018) développe une approche théorique originale en montrant que le financement participatif n'est réussi que si la plateforme, les porteurs de projet et la foule de contributeurs s'organisent partiellement en une organisation «co-dépendante ». En effet, selon l'auteur, le financement participatif mobilise des logiques diverses de réseaux, de communautés sociales, de marché mais aussi d'organisation. C'est pourquoi Nielsen fait appel à la notion d'«organisation partielle»d'Ahrne et Brunsson pour étudier les interactions et la coordination entre plateforme, porteurs de projets et contributeurs.

Ahrne et Brunsson (2010) ont développé la notion d'organisation partielle pour expliquer ce qui existe en dehors des organisations telles que les firmes, mais qui ne constitue ni une forme de réseau ni une forme d'institution. Au contraire, les deux sociologues suédois arguent que de nombreux phénomènes sociétaux relèvent d'un ordre social décidé mais incomplètement organisé. Une organisation complète, selon Ahrne et Brunsson, est définie comme un ordre social décidé combinant cinq éléments : des membres, une hiérarchie, un ensemble de règles, une surveillance du respect de ces règles et des sanctions. La combinaison sélective d'un ou plusieurs de ces éléments produit de l'organisation partielle (Ahrne, Brunsson, \& Seidl, 2016).

C'est précisément le cas du crowdlending : le processus de financement et les mécanismes de coordination des trois types d'acteurs (plateforme, foule et porteurs de projet) reposent sur une forme d'organisation partielle. Dans cette organisation partielle, les trois types d'acteurs prennent de façon incomplète des décisions sur les cinq composants identifiés par Ahrne et Brunsson : l'appartenance, la hiérarchie, les règles, la surveillance et les sanctions.

Dans la lignée des travaux récents ayant développé cette notion (Ahrne \& Brunsson, 2010; Grothe-Hammer, 2018; Järvi, Almpanopoulou, \& Ritala, 2018; Nielsen, 2018), les cinq éléments pré-cités donnent lieu à des décisions concrètes qui fixent les limites d'une organisation partielle :

1) Les décisions d'appartenance, c'est-à-dire le choix des membres de l'organisation et la façon dont ils y contribuent ou participent ; 
2) Les décisions sur la hiérarchie afin de définir quels organes ou acteurs détiennent un pouvoir central sur les autres dans l'organisation ;

3) Les décisions sur l'ensemble des règles ou paramètres qui régissent les interactions entre les acteurs et définissent des objectifs communs à atteindre ;

4) Les décisions sur la mise en place d'une surveillance du respect de ces règles, passant par l'existence ou non de système de comptabilité par exemple ;

5) Les décisions sur la mise en place de sanctions pour récompenser ou punir les membres qui respectent ou ne respectent pas les règles et les objectifs communs.

Ainsi, dans le modèle d'organisation partielle du crowdlending proposé par Nielsen, la plateforme a un droit de regard strict sur la sélection des porteurs de projet et leur appartenance à l'organisation partielle du financement participatif. Dans ce modèle, la hiérarchie fait souvent défaut ou se retrouve partagée entre la plateforme et les porteurs de projets. Ainsi le financement participatif, pris comme un écosystème d'acteurs interagissant, constitue une organisation partielle. La surveillance par les uns et les autres varie d'un acteur à l'autre. Si la foule ne prend pas de décision sur les règles définissant la participation de la plateforme, elle possède en revanche un pouvoir de surveillance et de sanctions strictes sur les porteurs de projet (voir figure 1). Certaines décisions, notamment du porteur de projet sur le choix de telle ou telle plateforme de financement, relèvent de ce que Nielsen appelle le «boundary pushing ». En mettant en compétition des plateformes de crowdfunding, le porteur de projet peut en effet déplacer la frontière de l'appartenance, c'est-à-dire affecter la participation d'une plateforme à l'organisation partielle. 
Figure 1 :

Définition d'une grille d'analyse « organisation partielle » appliquée au crowdfunding (schéma adapté de Nielsen, 2018, p.10)

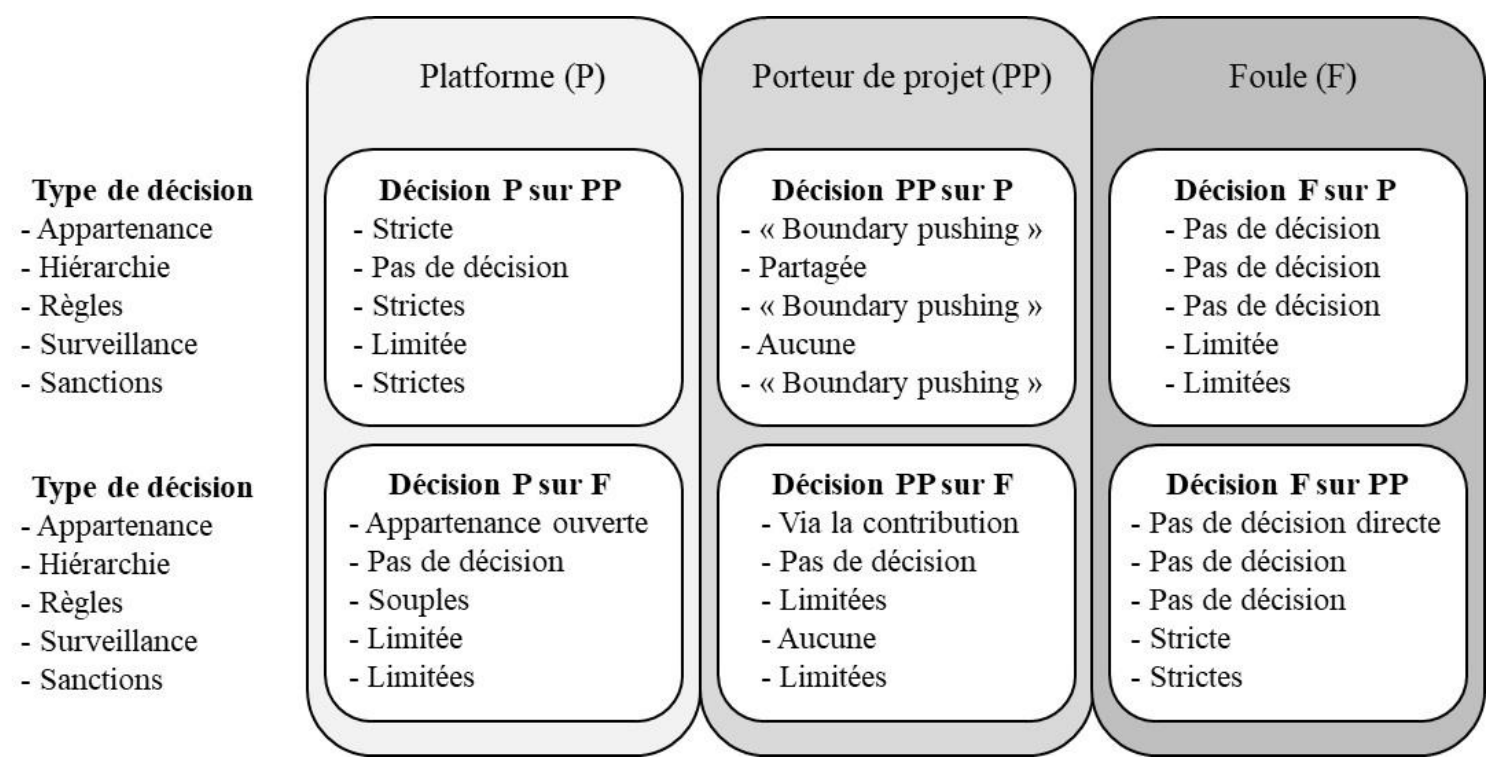

Il faut bien comprendre que dans le modèle de Nielsen, c'est le crowdlending luimême qui est étudié comme une organisation partielle. Il ne s'agit pas de la plateforme prise séparément par exemple. C'est l'ensemble des interactions et des décisions entre la plateforme, les porteurs de projet et la foule, qui est analysé comme une forme particulière d'organisation. Or Nielsen montre dans son analyse que les relations entre les trois types d'acteurs sont profondément interconnectées et interdépendantes. Sans cette inter- ou «co-dépendance » organisationnelle, l'auteur montre que le financement participatif est voué à l'échec. Si par exemple, la plateforme n'exerce pas son pouvoir strict de décision sur l'appartenance du porteur de projet, c'est-à-dire, si elle ne le sélectionne pas correctement, c'est tout le processus du financement participatif qui risque d'échouer.

Cependant, cette approche théorique néglige au moins deux aspects principaux du crowdlending: d'une part la diversité des fonctions de l'IC dans cette organisation partielle, et d'autre part le rôle de l'expertise sous la forme de l'EC. Pourtant, des travaux récents ont montré la place centrale que peut parfois jouer l'EC dans le financement participatif (Calme et al., 2018). La question est donc de savoir quel rôle l'expert-comptable peut jouer dans l'utilisation de l'intelligence collective dans le crowdlending et à quelles conditions. 
Nous nous intéressons en particulier à l'EC car le développement de nouveaux business models ou écosystèmes entrepreneuriaux dont fait partie le crowdlending soulève des enjeux importants par rapport au rôle de cette profession. Si de nombreuses études s'intéressent aux impacts des nouvelles technologies sur le contrôle de gestion (Ebondo \& Pigé, 2002; Meyssonnier, 2012; Trébucq, 2006), relativement peu s'intéressent à l'évolution du métier d'EC et à sa place dans une société «participative»s'appuyant sur l'IC. Or, l'EC demeure une figure incontournable, notamment dans la relation avec les TPE/PME (Chapellier, 2003; Vézina \& Fortin, 2002), une figure forcée de s'adapter sans cesse à un environnement en constante évolution (Cormier, Lapointe-Antunes, \& Magnan, 2012; Frey \& Osborne, 2017; Ndao \& Charles-Cargnello, 2015; Susskind \& Susskind, 2015).

\section{Méthodologie de la recherche}

Dans le but de mieux comprendre le rôle de l'EC dans l'expression de l'IC dans le crowdlending, nous avons mené une étude de cas approfondie. Afin d'explorer cette problématique dans un secteur émergent comme le crowdlending, la méthode de l'étude de cas parait la plus appropriée (Eisenhardt, 1989). Si de nombreux travaux s'intéressent à l'IC et ont amélioré notre compréhension de son expression dans le crowdfunding en général, peu de données permettaient jusqu'ici de préciser la place de l'EC dans l'expression de l'IC le crowdlending.

\subsection{Contexte empirique : l'échec du partenariat entre l'Ordre des experts- comptables et la plateforme Lendopolis}

Cet article s'intéresse à un partenariat signé en septembre 2014 par une plateforme de crowdlending, Lendopolis, et l'Ordre des experts-comptables (Calme et al., 2018). L'objectif de ce partenariat était de mettre à la disposition du public, pour chaque projet proposé au financement, des données financières robustes et attestées dans l'espoir que le public se saisisse de ces données, en débatte publiquement sur le forum et qu'émerge une forme d'IC dans la sélection des projets. Ce partenariat, qui n'a été appliqué que sur une période de 18 mois, a été vécu comme un échec par ses signataires. L'étude des échecs a un intérêt méthodologique puisqu'elle permet de mettre en évidence des mécanismes ou des caractéristiques difficilement identifiables par ailleurs et d'en proposer une amélioration théorique (Borins, 2001). 


\subsection{Collecte des données}

Notre collecte de données s'est organisée en trois grandes étapes (Voir Tableau 1). Dans une première phase exploratoire, nous avons commencé par rencontrer la plateforme Lendopolis afin d'avoir une première appréhension du fonctionnement et de la philosophie de cette plateforme dans le contexte français. C'est à ce moment que nous avons pris conscience de l'importance du partenariat avec l'Ordre des ExpertsComptables. Nous avons ensuite entamé la deuxième phase de la collecte de données en lançant une nouvelle campagne d'entretiens cherchant à faire émerger le fonctionnement pratique de l'IC chez Lendopolis et le rôle de son partenariat avec l'Ordre des Experts Comptables. Dans une troisième phase d'entretiens de consolidation, nous avons concentré notre attention sur les experts-comptables pour obtenir leurs retours d'expérience sur le partenariat et plus généralement sur le crowdlending. Tous nos entretiens, et ce tout au long des différentes phases, ont été enregistrés et retranscrits. Parallèlement, nous avons aussi collecté les données comptables et financières mises à la disposition de la foule sur tous les projets proposés sur Lendopolis ainsi que l'intégralité des échanges textuels sur les forums entre foule et porteurs de

projet. 


\section{Tableau 1 :}

\section{Synthèse de la collecte de données}

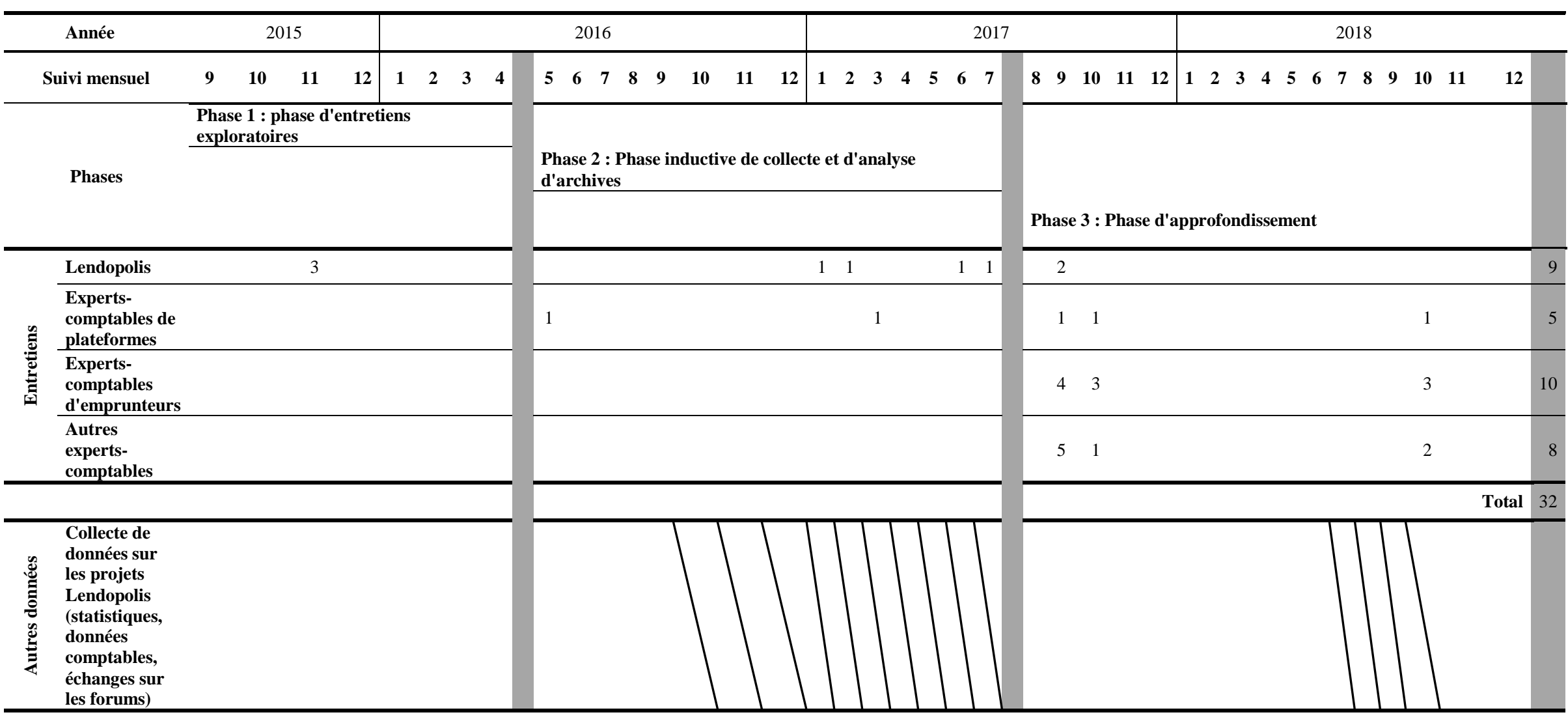




\subsection{Analyse des données}

Pour traiter ce riche matériau, nous avons d'abord adopté une analyse de contenu thématique (Miles, Huberman, \& Saldaña, 2013). Après la retranscription des entretiens, chaque auteur a lu séparément et à plusieurs reprises le matériau pour s'imprégner du cas (Dumez, 2016). Ensuite nous avons sélectionné puis extrait les verbatims et les textes les plus pertinents pour l'analyse de l'IC et de la place de l'EC.

Nous avons cherché à comprendre d'une part les fonctions de la foule dans le crowdlending, et d'autre part le rôle de l'EC dans cet «empowerment» de la foule. Pour ce faire, nous avons procédé en trois temps. Dans un premier temps, nous avons étudié le fonctionnement théorique du partenariat tel que formulé par Lendopolis avec l'Ordre des Experts-Comptables. A cette fin, nous avons cartographié les actions et les fonctions des différents acteurs dans l'organisation partielle du crowdlending: plateforme, porteurs de projet, foule et EC (Figure 2).

Dans un second temps, nous avons analysé la mise en pratique de ce fonctionnement théorique en identifiant les cas de réussite et d'échec de financement afin de mettre en évidence si ces échecs relevaient d'une incompétence de l'IC. Dans ce but, nous avons construit une base de données sur tous les projets passés ou en cours de remboursement de Lendopolis (215 projets). Pour tous ces projets nous avons recueilli les données mises à la disposition de la foule et l'intégralité des interactions sur les forums, là où s'exprime l'IC, en séparant les projets en six catégories : 1) projet remboursé, 2) en cours de remboursement, 3) projet frauduleux, 4) collecte échouée, 5) défaut de paiement, 6) retard de remboursement. Nous avons listé pour chaque projet les interactions financières qualifiées (portant sur les données attestées par 1'EC) entre la foule et le porteur de projet et catégorisées en : a) absence de réponse du porteur à une question de la foule, b) aberration relevée par la foule, c) réponse incompréhensible du porteur de projet, d) réponse intelligible du porteur de projet mais non vérifiée (voir tableau 2 ci-après).

Dans un troisième temps, nous avons appliqué le concept d'organisation partielle d'Ahrne et Brunsson (2010) et le modèle de Nielsen (2018) au cas du crowdlending chez Lendopolis. Dans la lignée de travaux récents s'appuyant sur les travaux d'Ahrne et Brunsson (Grothe-Hammer, 2018; Järvi et al., 2018), nous avons donc utilisé les concepts d'organisation partielle et d'organisation co-dépendante comme « sensitising 
concepts » (Järvi et al., 2018), c'est-à-dire comme une grille de lecture permettant une montée en généralité de notre analyse. Cela nous a permis d'identifier certains facteurs organisationnels pouvant expliquer l'échec du partenariat, mais aussi de repenser le fonctionnement du crowdlending (Figure 4) et développer un modèle théorique de codépendance élargie.

3. Le partenariat avec l'Ordre des Experts-Comptables : un détournement défectueux de l'organisation co-dépendante du financement participatif

Dans cette section nous décrivons le fonctionnement du partenariat et étudions ses échecs à la lumière du modèle d'organisation co-dépendante.

\subsection{Un fonctionnement visant à déléguer la prise de décisions à l'expert- comptable (EC) et à la foule}

Le partenariat entre Lendopolis et l'Ordre des experts-comptables a été construit pour répondre à deux principaux défis : 1) la bonne appréciation du risque de défaut des entreprises candidates à un financement, et 2) la mise en place d'un cadre permettant à l'IC de s'exprimer. Le fonctionnement de ce partenariat est résumé à la figure 2 : il repose sur une délégation à l'EC d'un pouvoir discrétionnaire sur l'acceptation d'un projet, et sur une délégation à la foule de la décision d'investissement. 


\section{Figure 2 :}

\section{Fonctionnement du crowdlending chez Lendopolis dans le cadre du partenariat avec l'Ordre des Experts-Comptables}

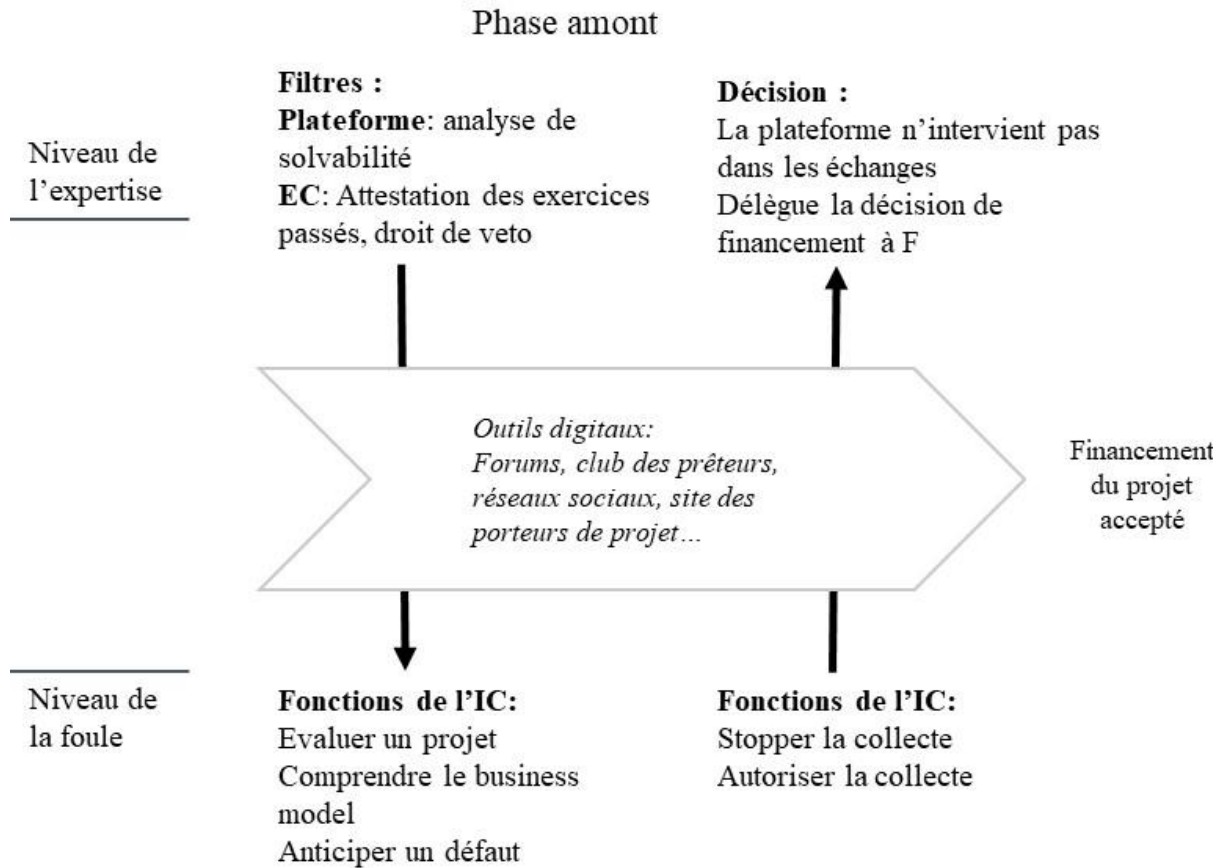

Phase aval

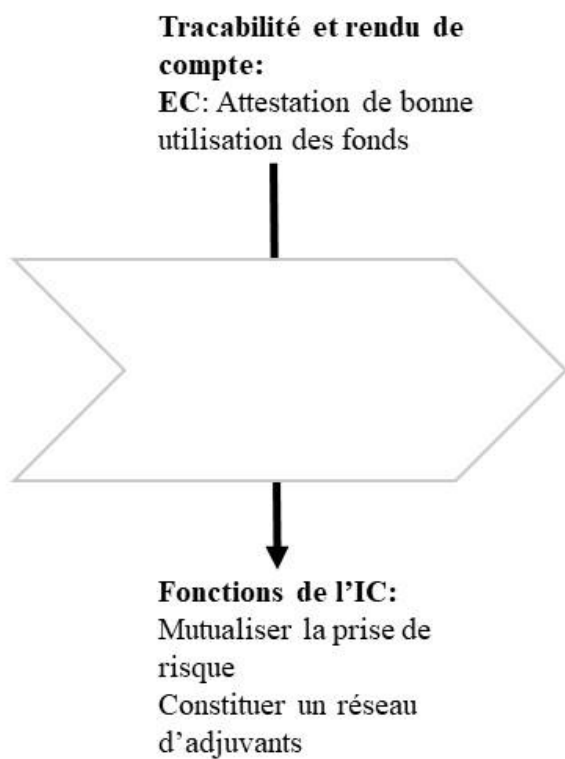

Il est difficile d'évaluer le risque de défaut d'une TPE, structure par nature fragile, et dont la soutenabilité financière est généralement extrêmement dépendante de l'action de son dirigeant. Les TPE sont par ailleurs dépourvues de contrôle interne, et les liasses fiscales qu'elles déposent ne permettent pas de saisir les dynamiques sous-jacentes à leur activité économique. Leurs prévisions d'activité doivent de surcroit être accueillies avec une certaine prudence :

"La plupart des PME [...] n'ont pas de DAF. Donc le mec, [dans] son prévisionnel [...], il peut mettre potentiellement n’importe quoi. » [Lendopolis]

L'EC, parce qu'il est le premier, et parfois le seul conseil des dirigeants de TPE est apparu à Lendopolis comme étant un interlocuteur à même de l'aider à relever le défi de l'appréciation du risque de défaut des TPE candidates au financement. L'EC pouvait également, par son intervention, fournir un cadre structurant à l'expression de l'IC sur la plateforme.

Les fondateurs de Lendopolis ont conçu leur projet de partenariat non pas d'abord comme un projet financier, mais comme un projet philosophique. Et au cœur de cette 
philosophie se trouve le concept d'IC selon lequel une foule de contributeurs particuliers peut exprimer une forme d'intelligence supérieure à même d'améliorer la sélection des projets en amont des campagnes de financement et de participer en aval à leur développement ou bien de supporter le coût d'un défaut de manière relativement indolore.

« Notre vraie innovation elle est dans l'intelligence collective. C'est parce qu'il y a une foule qui arrive sur un projet et que cette foule est plus intelligente qu'un analyste, qu'on va savoir si un projet est à risque ou pas. » [Lendopolis]

Le fonctionnement du partenariat, que résume la figure 2, introduit l'intervention d'un EC à deux étapes dans le processus d'investissement dans un projet : 1) une attestation obligatoire du prévisionnel par l'EC de l'entreprise, assortie, et c'est une découverte issue du terrain, d'un droit de veto informel et non public de l'EC sur la mise en ligne du projet, et 2) une attestation optionnelle de bonne utilisation des fonds collectés sur la plateforme. En devant valider l'atterrissage de l'année en cours et la cohérence des trois années du prévisionnel, l'EC engage sa signature sur le prévisionnel.

«Il y a quand même une labellisation morale en fait » [Lendopolis]

Par ailleurs, quand le prévisionnel est attesté, celui-ci permet d'indiquer explicitement les zones de risques du projet et de communiquer à la foule de potentiels contributeurs des données financières robustes. Celles-ci doivent servir de base au jeu de questions réponses publiques sur le forum avec le porteur de projet (figure 2).

Pour Lendopolis, l'EC a la possibilité de refuser d'attester un prévisionnel. Mais la plateforme considère que l'EC n'y serait pas incité car ce refus pourrait mettre en danger sa relation commerciale avec son client. Par ailleurs le prévisionnel ne permettrait pas à l'EC de transmettre à la foule toutes les informations dont il dispose sur le projet et sur son porteur. C'est pourquoi, Lendopolis a intégré à ses procédures de sélection la possibilité d'un droit de veto informel de l'EC sur la mise en ligne des projets portés par ses clients

«Dans le schéma de la machine, l'expert-comptable, qui a souvent un historique relationnel avec le dirigeant de l'entreprise a, lors de nos échanges, [...] un droit de veto. [...] Et sans mettre en danger la relation commerciale entre l'expertcomptable et son client parce que si l'expert-comptable met son veto, c'est la 
plateforme qui endosse, qui assume aux yeux du client cette décision. ” [Lendopolis]

Pour Lendopolis, il s'agirait, à travers ce droit de veto secret, de permettre à 1'EC de transmettre une partie de l'information implicite qu'il a acquise dans le cadre d'une relation de long terme avec son client quand cette information n'a pas pu explicitement être intégrée au prévisionnel, soit que cette information soit extra-financière, soit que, pour préserver sa relation commerciale avec son client, l'EC n'ait pas souhaité ou pas pu intégrer cette information au prévisionnel (hypothèse défendue par Lendopolis).

Au final, Lendopolis délègue à l'EC un pouvoir discrétionnaire informel et secret d'écarter un projet sans avoir besoin d'autres justifications que celle du libre exercice de son jugement professionnel.

"C'est lui qui a en main tous les éléments. Soit il a confiance, soit il n'a pas confiance. C'est du rouge ou du vert » [Lendopolis]

Ce droit de veto, qui n'apparait officiellement nulle part, a bel et bien été intégré systématiquement à la procédure de sélection des projets pendant les 18 mois qu'a duré le partenariat.

Enfin, par les interactions sur les forums entre foule et porteurs de projets, l'IC des contributeurs a pour fonctions d'évaluer les projets, d'en comprendre le business model, et d'anticiper les risques de défauts. La foule peut dès lors, seule, choisir de stopper la collecte, ou de l'autoriser, en théorie.

\subsection{L'échec de la double délégation}

Dans sa mise en application, le fonctionnement du partenariat a achoppé sur deux obstacles : un rejet massif du partenariat par les experts-comptables et une exploitation inaboutie de l'IC par la plateforme.

Tout d'abord, si le droit de veto est apparu à Lendopolis comme un moyen astucieux de mettre en valeur les compétences des experts-comptables tout en protégeant leurs relations commerciales, en réalité, à travers ce dispositif non-officiel Lendopolis a méconnu les exigences déontologiques et éthiques d'une profession réglementée, marquée du sceau de l'intérêt général. Loin de mobiliser la profession, ce dispositif l'a fortement braquée. Ce dispositif a été perçu comme dangereux et 
vexatoire. Les EC ont refusé de se voir déléguer ce droit de veto informel et secret qui n'était d'ailleurs pas officiellement prévu dans le texte du partenariat.

"Je suis très mal à l'aise avec ce droit de veto. Déontologiquement, c'est quand même très limite, non? Je ne me sens pas valorisé par ce droit de veto. Au contraire. De quel droit je dirais « stop » à un projet, en catimini, au téléphone avec quelqu'un qui n'est même pas expert-comptable, sans le dire à mon client qui me paie et vis-à-vis de qui j'ai des devoirs déontologiques ?»[Expertcomptable]

"Ce n'est pas pour rien que le droit de veto est implicite et qu'on ne le voit officiellement nulle part. C'est tout simplement illégal et contraire à notre déontologie. Dire vert sur un prévisionnel transmis au client et rouge au téléphone sans que le client soit informé... » [Expert-comptable]

En outre, ce droit de veto s'inscrivait pour Lendopolis dans une logique low cost de délégation de la sélection des projets à une profession réglementée, sans le dire officiellement, et sans la rémunérer d'aucune façon.

Au-delà du droit de veto, c'est l'IC qui n'est qu'incomplètement exploitée par la plateforme. Certes, les éléments financiers et les attestations de prévisionnels fournis par les experts-comptables sont effectivement utilisés par les contributeurs pour engager un dialogue public avec le porteur de projet. Ainsi des questions financières extrêmement précises et pertinentes sont posées :

«-Je suis surpris d'un tel montant de panier moyen car je ne comprends pas comment on peut passer commande de 800 Euros d'un coup pour des compléments alimentaires [Question d'un internaute]

-Notre panier moyen est en effet élevé car il s'agit de programmes dont la durée moyenne est de 8 mois» [Réponse du porteur de projet, catégorie réponse intelligible non vérifiée]

Le tableau 2 montre que les internautes se sont effectivement saisis des informations attestées par les EC pour initier des échanges avec les porteurs de projets. Ces échanges produisent une forme d'IC, i.e. des interactions sur les forums générant de l'information pouvant servir à la décision d'investissement. Ainsi, 11 cas sur 15 
d'interactions avec une réponse incompréhensible du porteur de projet à une question des contributeurs s'accompagnent d'un échec de collecte : la foule choisit donc de ne pas investir sur des signaux négatifs émis par le porteur de projet.

Tableau 2 :

Synthèse des interactions Foule-Porteur de projet à partir des informations attestées par les experts-comptables

\begin{tabular}{|c|c|c|c|c|c|c|}
\hline $\begin{array}{l}\text { Type de projet / } \\
\text { Type d'interaction }\end{array}$ & $\begin{array}{l}\text { Absence de } \\
\text { réponse à } \\
\text { une question } \\
\text { pertinente }\end{array}$ & $\begin{array}{l}\text { Aberration } \\
\text { financière ou } \\
\text { comptable } \\
\text { relevée par la } \\
\text { foule }\end{array}$ & $\begin{array}{c}\text { Réponse } \\
\text { incompréhensible } \\
\text { ou hors sujet du } \\
\text { porteur de projet }\end{array}$ & $\begin{array}{c}\text { Réponse } \\
\text { intelligible } \\
\text { du porteur de } \\
\text { projet mais } \\
\text { vérifiée }\end{array}$ & $\begin{array}{c}\text { Total } \\
\text { d'interactions } \\
\text { financières } \\
\text { qualifiées }\end{array}$ & $\begin{array}{c}\text { Nombre } \\
\text { de } \\
\text { projets }\end{array}$ \\
\hline Projets remboursés & 4 & 1 & 0 & 13 & 18 & 20 \\
\hline $\begin{array}{l}\text { Projets en cours de } \\
\text { remboursement }\end{array}$ & 13 & 6 & 0 & 39 & 58 & 141 \\
\hline Projets Frauduleux & 1 & 1 & 0 & 0 & 2 & 2 \\
\hline Collectes échouées & 1 & 1 & 11 & 0 & 13 & 8 \\
\hline Défauts de paiement & 1 & 4 & 4 & 16 & 25 & 32 \\
\hline Retards de Paiement & 2 & 0 & 0 & 6 & 8 & 12 \\
\hline Total & 22 & 13 & 15 & 74 & 124 & 215 \\
\hline
\end{tabular}

Cependant, la plateforme estime que la foule doit, d'elle-même, décider seule d'interrompre ou non une collecte. La foule s'appuie sur des données attestées mais elle est livrée à elle-même et ne bénéficie d'aucun soutien dans sa discussion avec le porteur de projet. Le porteur de projet peut donc aisément la tromper.

Par exemple, dans un cas d'interaction avec réponse intelligible $d u$ PP non vérifiée, un particulier intervient sur le forum et s'interroge sur le poste créance client qui a fortement augmenté en 2015. Il demande à ce que soient communiqués le délai de règlement moyen des clients ainsi que le montant des créances clients dont l'antériorité est supérieure à un an ou qui pourraient être considérées comme douteuses. Le porteur de projet répond que le délai de règlement des clients, s'il était important fin 2015, a été divisé par deux depuis, passant de 120 à 60 jours, et qu'il n'existe aucune créance douteuse ni facture impayée ayant fait l'objet d'une procédure particulière. Or, sa réponse n'est ni vérifiée par la plateforme, ni par personne. Les contributeurs le croient et financent entièrement le projet. Deux mois après, le projet fait défaut et son porteur révèle alors que 123000 euros de créances impayées le poussent à arrêter son activité.

Plus généralement, dans 16 cas, l'absence de contrôle par la plateforme des réponses apportées à la foule par le porteur de projet s'accompagne de défauts de paiements. De manière plus flagrante encore, dans 4 cas sur 13, l'identification par la 
foule d'une aberration financière ou comptable est corrélée à un défaut de paiement du porteur de projet.

Ainsi l'IC qui s'exprime sur les forums n'est ni robuste ni exploitée. En effet, l'information que produit l'IC sur les forums ne fait l'objet d'aucun contrôle ni par la plateforme ni par l'EC, qui n'est de toute façon pas mandaté par elle sur le forum. Il n'y a pas ici, sur le forum, d'interaction entre foule et experts, quels qu'ils soient. La plateforme ne surveille pas les échanges, ne les prend pas en compte et n'en tire pas les conséquences qui pourraient s'imposer. Notamment, les aberrations financières relevées par la foule ne donnent lieu à aucune réaction de la plateforme alors qu'elle aurait pu choisir d'interrompre la collecte.

\subsection{Des altérations du modèle d'organisation co-dépendante}

Le fonctionnement de Lendopolis dans son partenariat avec l'Ordre des ExpertsComptables a introduit certaines altérations au modèle d'organisation co-dépendante à la Nielsen, comme le résume la figure 3. Nous expliquons comment ces altérations ont pu contribuer à l'échec du partenariat.

Figure 3 :

Le fonctionnement de Lendopolis au prisme du modèle d'organisation codépendante (en italique les altérations par rapport au modèle de Nielsen)

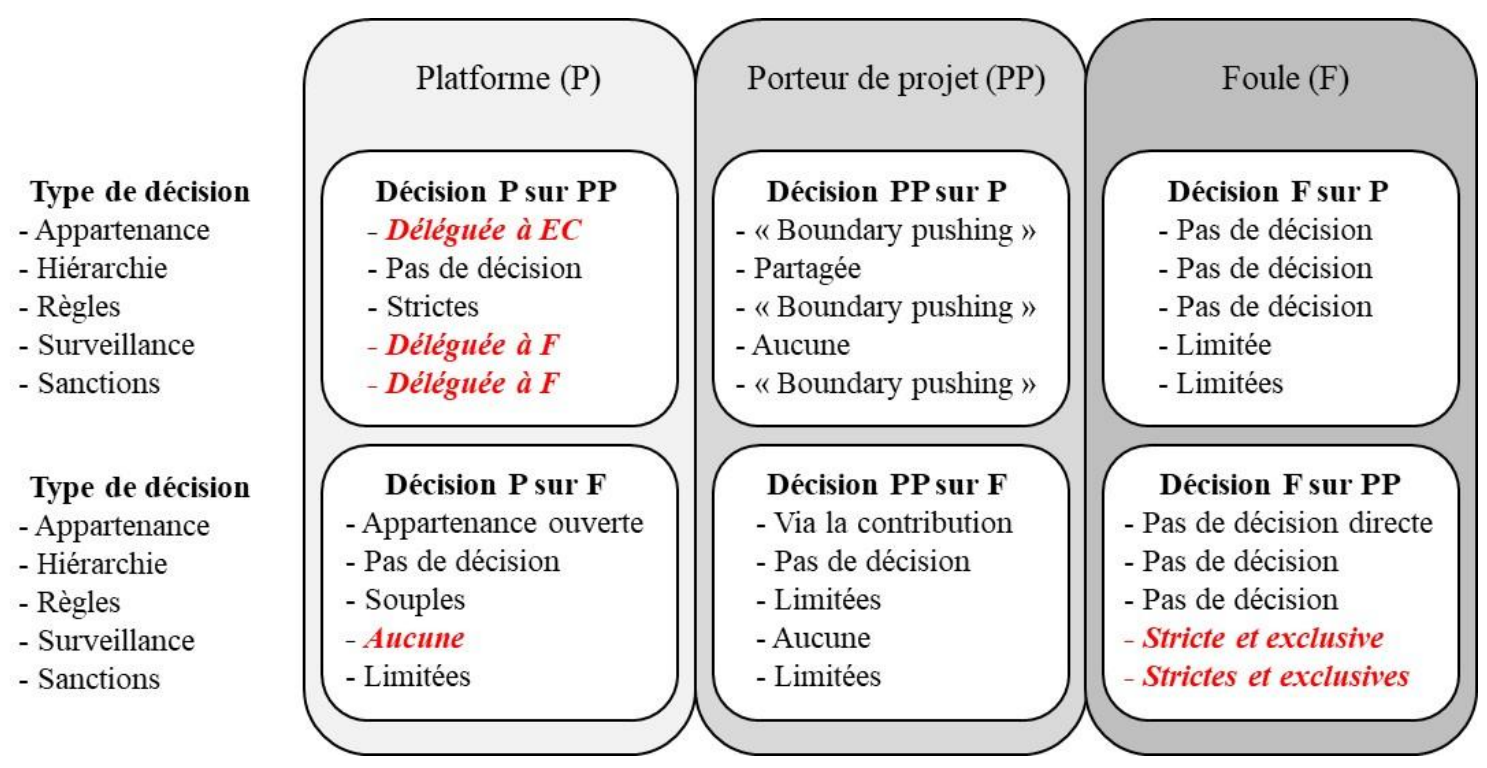


Les tableaux $3 \mathrm{a}$ à $3 \mathrm{c}$ reviennent plus en détail sur les altérations du modèle d'organisation co-dépendante en étudiant pour chaque aspect, le pouvoir de décision organisationnelle (i.e. sur l'appartenance, la hiérarchie, les règles, la surveillance et les sanctions) d'un acteur sur l'autre : plateforme, porteur de projet, foule. Rappelons que ces décisions portent sur le financement participatif comme une organisation partielle rassemblant plateforme, porteurs de projet et foule.

\section{Tableau 3a :}

Comparaison des décisions organisationnelles de la plateforme $(P)$ sur les porteurs de projet (PP) entre le modèle de Nielsen et le cas Lendopolis

\begin{tabular}{lll}
\hline $\begin{array}{l}\text { Type de } \\
\text { décision }\end{array}$ & P sur PP(Nielsen) & P sur PP (Lendopolis) \\
\hline Appartenance & Stricte & Déléguée à l'EC sur la sélection des projets \\
\hline Hiérarchie & Pas de décision & Pas de décision \\
\hline Règles & Strictes & Strictes \\
\hline Surveillance & Limitée & Déléguée pendant la campagne à la foule \\
\hline Sanctions & Strictes & Déléguées pendant la campagne à la foule \\
\hline
\end{tabular}

Tableau 3b :

Comparaison des décisions organisationnelles de la plateforme (P) sur la foule (F), Nielsen/Lendopolis

\begin{tabular}{lll}
\hline $\begin{array}{l}\text { Type de } \\
\text { décision }\end{array}$ & P sur F (Nielsen) & P sur F (Lendopolis) \\
\hline Appartenance & $\begin{array}{l}\text { Appartenance } \\
\text { ouverte }\end{array}$ & Appartenance ouverte \\
\hline Hiérarchie & Pas de décision & Pas de décision \\
\hline Règles & Souples & Souples \\
\hline Surveillance & Limitée & Aucune \\
\hline Sanctions & Limitées & Limitées \\
\hline
\end{tabular}

\section{Tableau 3c :}

Comparaison des décisions organisationnelles de la foule (F) sur les porteurs de projet (PP), Nielsen/Lendopolis

\begin{tabular}{lll}
\hline $\begin{array}{l}\text { Type de } \\
\text { décision }\end{array}$ & F sur PP (Nielsen) & F sur PP(Lendopolis) \\
\hline Appartenance & $\begin{array}{l}\text { Pas de décision } \\
\text { directe }\end{array}$ & Pas de décision directe \\
\hline Hiérarchie & Pas de décision & Pas de décision \\
\hline Règles & Pas de décision & Pas de décision \\
\hline Surveillance & Stricte & Stricte et exclusive \\
\hline Sanctions & Strictes & Strictes et exclusives \\
\hline
\end{tabular}


Comme indiqué au tableau 3a, la plateforme s'écarte du modèle de Nielsen en délégant un pouvoir de décision sur les porteurs de projets : le pouvoir de décision sur l'appartenance des porteurs de projets est délégué à l'EC, et le pouvoir de décision sur la surveillance et les sanctions des porteurs de projets (PP) est délégué à la foule. L'influence de la plateforme sur le PP en termes d'appartenance est stricte chez Nielsen car la plateforme conduit en amont de la mise en ligne une première étude de la solvabilité des projets via des algorithmes de scoring et des KYC. Ces vérifications sont nécessaires selon Nielsen afin de prévenir les fraudes. En revanche, même si Lendopolis fait elle-même une première analyse de solvabilité des projets, cette plateforme a demandé à l'EC de prendre en charge la décision d'appartenance, non seulement via l'attestation de prévisionnel, mais surtout via le droit de veto informel que l'EC peut actionner arbitrairement et sans en informer le porteur de projet. Or, ce droit de veto ne fonctionne pas, comme mentionné précédemment, pour au moins deux raisons, une raison liée à une incompatibilité déontologique de mise en œuvre de ce dispositif par les experts-comptables et une raison liée à l'absence de rémunération de ce service par la plateforme.

Le tableau 3 b montre, quant à lui, que la plateforme Lendopolis n'exerce aucun contrôle sur la foule, tandis que dans le modèle de Nielsen ce contrôle existe même s'il est limité. Enfin le tableau 3c, sorte de miroir du tableau 3a, révèle que les principales altérations du modèle de Nielsen portent sur la surveillance et la sanction. C'est en effet la foule qui exerce un pouvoir strict et exclusif sur la surveillance et la sanction des porteurs de projet, puisque Lendopolis lui a délégué ce pouvoir (3a). Lendopolis n'intervient en effet nullement sur le forum, et s'interdit de mettre fin de son propre chef à une collecte en cours.

$\mathrm{Au}$ final, Lendopolis a introduit plusieurs déséquilibres dans le modèle de codépendance : 1) la plateforme fait intervenir l'EC comme un nouvel acteur à part entière dont les actions ne sont pas co-dépendantes aux autres acteurs, 2) la plateforme délègue un pouvoir décisionnaire à l'EC et à la foule, 3) elle ne contrôle ni l'EC, ni le porteur de projet, ni la foule.

\section{Vers un modèle élargi d'organisation co-dépendante incluant l'Expert- Comptable (EC)}


A partir de cette description et du modèle de Nielsen, il est possible de définir un nouveau modèle de co-dépendance dans le financement participatif en s'appuyant sur une quatrième figure, celle de l'EC. Pour ce faire, nous proposons de revisiter le processus du financement participatif appliqué chez Lendopolis (Figure 4) à partir de l'analyse que nous en avons faite, des entretiens avec les experts-comptables, des interactions entre foule et porteurs de projet et du modèle de Nielsen. Dans ce nouveau processus, le droit de veto de l'EC disparait. Le filtre des porteurs de projet repose désormais sur des analyses de solvabilité et autres due diligences, ainsi que sur l'attestation des exercices passés et du prévisionnel par l'EC.

\section{Figure 4 :}

\section{Proposition de fonctionnement revisité du crowdlending}

Phase amont

\section{Filtres :}

P: analyse de solvabilité

Niveau de

l'expertise

\section{EC: Attestation des exercices}

passés et du prévisionnel

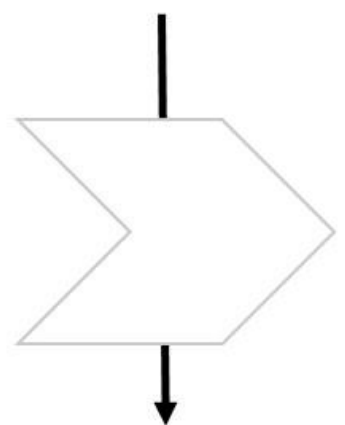

Niveau de la foule

$$
\begin{aligned}
& \text { Fonctions de l'IC: } \\
& \text { Evaluer un projet } \\
& \text { Comprendre le business } \\
& \text { model } \\
& \text { Anticiper un défaut }
\end{aligned}
$$

Phase aval

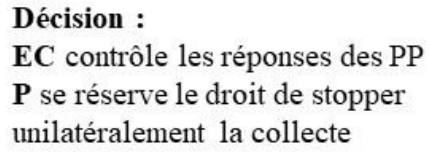

Tracabilité et rendu de compte:

EC: Attestation de bonne utilisation des fonds

L'IC a toujours les mêmes fonctions en amont et en aval de la collecte, permises notamment par l'EC : évaluer un projet, comprendre le business models, mutualiser les risques. En revanche, dans ce modèle revisité, nous proposons que l'EC joue un rôle de contrôle sur les forums. Afin de consolider et d'activer l'IC, il est en effet apparu nécessaire qu'une surveillance des interactions entre foule et porteurs de projet soit effectuée. Il s'agit ici de vérifier la cohérence et la fidélité à la réalité des réponses faites à la foule par le porteur de projet. Il s'agit également de remonter à la plateforme les informations nouvelles issues du forum et susceptibles de remettre en cause l'analyse initiale de solvabilité. Il semble judicieux qu'un ou plusieurs EC mandatés par la 
plateforme effectuent ce contrôle en lien avec l'EC du porteur de projet. Cela implique une forme ou une autre d'intéressement (recrutement par la plateforme, sous-traitance ou autre forme de rémunération). Enfin en aval, l'EC conserve la même fonction d'attestation de bonne utilisation des fonds afin d'assurer la traçabilité et le rendu de comptes.

Ce rôle prépondérant de l'EC constitue cependant une altération du modèle de Nielsen qu'il convient à présent de conceptualiser, d'analyser et d'intégrer dans le modèle de co-dépendance (synthétisé à la Figure 5).

Figure 5 :

Modèle revisité de co-dépendance entre plateforme-porteurs de projet-fouleexpert-comptable (EC) (mis en évidence : nos propositions)

\begin{tabular}{|c|c|c|c|c|}
\hline & Platforme (P) & Porteur de projet (PP) & Foule (F) & Expert-Comptable (EC) \\
\hline $\begin{array}{l}\text { Type de décision } \\
\text { - Appartenance } \\
\text { - Hiérarchie } \\
\text { - Règles } \\
\text { - Surveillance } \\
\text { - Sanctions }\end{array}$ & $\begin{array}{l}\text { Décision P sur PP } \\
\text { - Stricte, partagée } \\
\text { - Pas de décision } \\
\text { - Strictes } \\
\text { - Stricte, partagée } \\
\text { - Strictes, partagées }\end{array}$ & $\begin{array}{l}\text { Décision PPsur P } \\
\text { - Boundary pushing } \\
\text { - Partagée } \\
\text { - Boundary pushing } \\
\text { - Aucune } \\
\text { - Boundary pushing }\end{array}$ & $\begin{array}{l}\text { Décision F sur P } \\
\text { - Pas de décision } \\
\text { - Pas de décision } \\
\text { - Pas de décision } \\
\text { - Limitée } \\
\text { - Limitées }\end{array}$ & $\begin{array}{l}\text { Décision EC sur P } \\
\text { - Boundary pushing } \\
\text { - Pas de décision } \\
\text { - Strictes } \\
\text { - Aucune } \\
\text { - Boundary pushing }\end{array}$ \\
\hline $\begin{array}{l}\text { Type de décision } \\
\text { - Appartenance } \\
\text { - Hiérarchie } \\
\text { - Règles } \\
\text { - Surveillance } \\
\text { - Sanctions }\end{array}$ & $\begin{array}{l}\text { Décision P sur F } \\
\text { - Appartenance ouverte } \\
\text { - Pas de décision } \\
\text { - Souples } \\
\text { - Stricte, partagée } \\
\text { - Sanctions limitées }\end{array}$ & $\begin{array}{l}\text { Décision PPsur F } \\
\text { - Via la contribution } \\
\text { - Pas de décision } \\
\text { - Limitées } \\
\text { - Aucune } \\
\text { - Limitées }\end{array}$ & $\begin{array}{l}\quad \text { Décision F sur PP } \\
\text { - Pas de décision directe } \\
\text { - Pas de décision } \\
\text { - Souples } \\
\text { - Stricte, partagée } \\
\text { - Strictes, partagées }\end{array}$ & $\begin{array}{l}\text { Décision EC sur PP } \\
\text { - Stricte et partagée } \\
\text { - Pas de décision } \\
\text { - Strictes } \\
\text { - Stricte, partagée } \\
\text { - Aucune }\end{array}$ \\
\hline $\begin{array}{l}\text { Type de décision } \\
\text { - Appartenance } \\
\text { - Hiérarchie } \\
\text { - Règles } \\
\text { - Surveillance } \\
\text { - Sanctions }\end{array}$ & $\begin{array}{l}\text { Décision P sur EC } \\
\text { - Aucune, ou stricte } \\
\text { - Pas de décision } \\
\text { - Strictes } \\
\text { - Aucune } \\
\text { - Aucune }\end{array}$ & $\begin{array}{l}\text { Décision PP sur EC } \\
\text { - Boundary pushing } \\
\text { - Pas de décision } \\
\text { - Boundary pushing } \\
\text { - Aucune } \\
\text { - Boundary pushing }\end{array}$ & $\begin{array}{l}\text { Décision F sur EC } \\
\text { - Pas de décision } \\
\text { - Partagée } \\
\text { - Pas de décision } \\
\text { - Pas de décision } \\
\text { - Pas de décision }\end{array}$ & $\begin{array}{l}\text { Décision EC sur F } \\
\text { - Pas de décision } \\
\text { - Partagée } \\
\text { - Pas de décision } \\
\text { - Stricte, partagée } \\
\text { - Aucune }\end{array}$ \\
\hline
\end{tabular}

Le modèle que nous proposons de co-dépendance élargie intègre ainsi l'EC comme quatrième acteur à part entière. Pour chaque décision, nous présentons donc soit un retour à Nielsen, soit une transformation du modèle de Lendopolis, comme le synthétisent les tableaux $4 \mathrm{a}$ et $4 \mathrm{~b}$. 
Tableau 4a :

\section{Comparaison des décisions dans les trois approches}

\begin{tabular}{|c|c|c|c|}
\hline $\begin{array}{l}\text { Type de } \\
\text { décision }\end{array}$ & $\begin{array}{l}\text { P sur PP } \\
\text { Nielsen }\end{array}$ & P sur PP Lendopolis & P sur PP Nielsen revisité \\
\hline Appartenance & Stricte & $\begin{array}{l}\text { Déléguée à l'EC sur la } \\
\text { sélection, et dans les faits, } \\
\text { droit de rétractation } \\
\text { jamais utilisé par } \\
\text { Lendopolis }\end{array}$ & Stricte et partagée avec EC \\
\hline Hiérarchie & Pas de décision & Pas de décision & Pas de décision \\
\hline Règles & Strictes & Strictes & Strictes \\
\hline Surveillance & Limitée & $\begin{array}{l}\text { Déléguée pendant la } \\
\text { campagne à la foule }\end{array}$ & $\begin{array}{l}\text { Stricte, partagée avec EC } \\
\text { et } F\end{array}$ \\
\hline Sanctions & Strictes & $\begin{array}{l}\text { Déléguées pendant la } \\
\text { campagne à la foule }\end{array}$ & Strictes, partagées avec $F$ \\
\hline $\begin{array}{l}\text { Type de } \\
\text { décision }\end{array}$ & P sur F Nielsen & P sur F Lendopolis & P sur F Nielsen revisité \\
\hline Appartenance & $\begin{array}{l}\text { Appartenance } \\
\text { ouverte }\end{array}$ & Appartenance ouverte & Appartenance ouverte \\
\hline Hiérarchie & Pas de décision & Pas de décision & Pas de décision \\
\hline Règles & Souples & Souples & Souples \\
\hline Surveillance & Limitée & Aucune & Stricte, partagée avec $\mathrm{EC}$ \\
\hline Sanctions & Limitées & Limitées & Limitées \\
\hline $\begin{array}{l}\text { Type de } \\
\text { décision }\end{array}$ & $\begin{array}{l}\text { F sur PP } \\
\text { Nielsen }\end{array}$ & F sur PP Lendopolis & F sur PP Nielsen revisité \\
\hline Appartenance & $\begin{array}{l}\text { Pas de décision } \\
\text { directe }\end{array}$ & Pas de décision directe & Pas de décision directe \\
\hline Hiérarchie & Pas de décision & Pas de décision & Pas de décision \\
\hline Règles & Pas de décision & Pas de décision & Souples \\
\hline Surveillance & Stricte & Stricte et exclusive & $\begin{array}{l}\text { Stricte et partagée avec } E C \\
\text { et } P\end{array}$ \\
\hline Sanctions & Strictes & Strictes et exclusives & Strictes et partagées avec $P$ \\
\hline
\end{tabular}

Comme expliqué précédemment, le droit de veto disparait car il produit un découplage entre discours et pratiques et ne respecte pas la déontologie de la profession. En revanche, l'EC doit attester les exercices passés et le prévisionnel. Un EC qui ne fournirait pas ces attestations conduirait de fait le PP à ne pas être accepté sur la plateforme. Ainsi le pouvoir de décision sur l'appartenance du PP dans ce modèle revisité est partagé entre $\mathrm{P}$ et EC. Autre distinction que nous apportons, la plateforme effectue un contrôle strict des PP, contrôle partagé avec la foule et l'EC. De même, P et EC effectuent un contrôle partagé des échanges sur les forums entre F et PP afin d'une part d'activer l'IC, et d'autre part d'être en mesure d'arrêter une collecte même si la foule serait disposée à financer le projet.

\section{Tableau 4b :}


Intégration de l'EC dans le modèle de co-dépendance

\begin{tabular}{|c|c|}
\hline Type de décision & EC sur $P$ \\
\hline Appartenance & «Boundary pushing » \\
\hline Hiérarchie & Pas décision \\
\hline Règles & $\begin{array}{l}\text { Règles strictes, (être enregistrée à l'ORIAS, respecter le cadre } \\
\text { règlementaire, avoir un statut légal IFP, CIP ou PSI) }\end{array}$ \\
\hline Surveillance & Aucune \\
\hline Sanctions & «Boundary pushing » sur l'appartenance \\
\hline Type de décision & EC sur PP \\
\hline Appartenance & Stricte et partagée avec $\mathrm{P}$ \\
\hline Hiérarchie & Pas de décision \\
\hline Règles & Règles strictes (le PP ne doit pas mentir sur les données financières etc) \\
\hline Surveillance & Surveillance stricte et partagée avec $\mathrm{F}$ et $\mathrm{P}$ pendant la campagne \\
\hline Sanctions & $\begin{array}{l}\text { Aucune (ce n'est pas l'EC qui prend la décision d'interrompre la } \\
\text { collecte) }\end{array}$ \\
\hline Type de décision & EC sur $\mathbf{F}$ \\
\hline Appartenance & Pas de décision \\
\hline Hiérarchie & $\begin{array}{l}\text { Hiérarchie partagée car EC fournit de l'information à F. F décide à partir } \\
\text { de cette information, il y a donc création de hiérarchies multiples. }\end{array}$ \\
\hline Règles & Pas de décision \\
\hline Surveillance & Surveillance stricte et partagée avec $\mathrm{P}$ sur les forums \\
\hline Sanctions & Aucune \\
\hline Type de décision & P sur EC \\
\hline Appartenance & $\begin{array}{l}\text { Pas de décision sur l'appartenance si c'est l'EC du PP ou stricte si c'est } \\
\text { un EC engagé par P }\end{array}$ \\
\hline Hiérarchie & Pas de décision \\
\hline Règles & $\begin{array}{l}\text { Strictes (l'EC doit attester les exercices passés et la bonne utilisation des } \\
\text { fonds, ainsi que contrôler les échanges avec la foule) }\end{array}$ \\
\hline Surveillance & Aucune \\
\hline Sanctions & Aucune \\
\hline Type de décision & PP sur EC \\
\hline Appartenance & Boundary pushing, l'EC du PP participe \\
\hline Hiérarchie & Pas de décision \\
\hline Règles & Boundary pushing \\
\hline Surveillance & Aucune \\
\hline Sanctions & Boundary pushing \\
\hline Type de décision & F sur EC \\
\hline Appartenance & Pas de décision \\
\hline Hiérarchie & $\begin{array}{l}\text { Hiérarchie partagée avec EC et } \mathrm{P}: \mathrm{F} \text { produit de l'information contrôlée } \\
\text { par EC à partir desquelles } \mathrm{P} \text { prend des décisions. Il y a donc hiérarchies } \\
\text { multiples }\end{array}$ \\
\hline Règles & Pas de décision \\
\hline Surveillance & Pas de décision \\
\hline Sanctions & Pas de décision \\
\hline
\end{tabular}


Similairement au cas PP sur P, l'EC a un pouvoir de décision sur la participation de la plateforme (son appartenance à l'organisation partielle qu'est le processus de crowdlending) de type «boundary pushing ». L'EC peut en effet conseiller au PP d'aller sur une plateforme concurrente. En conséquence, la décision sur la sanction est elle-même de forme « boundary pushing » (changement de plateforme).

$\mathrm{Au}$ final, comme l'indiquent les tableaux $4 \mathrm{a}$ et $4 \mathrm{~b}$, la co-dépendance entre plateforme, porteur de projet, foule et EC se traduit par un partage de certaines décisions organisationnelles, notamment sur l'appartenance, la surveillance et les sanctions du PP. L'objectif de ce modèle de co-dépendance élargie est double : 1) offrir une place plus grande à l'EC mais acceptable déontologiquement et financièrement, 2) exploiter l'IC en l'activant grâce aux contrôles de l'EC et de la plateforme sur les forums.

\section{Discussion des résultats}

Cet article a cherché à comprendre le rôle de l'EC dans l'exploitation de l'IC dans le crowdlending, ainsi que les conditions de l'intégration de l'EC dans le modèle d'organisation co-dépendante. Pour ce faire, nous avons mené une étude de cas approfondie du fonctionnement de Lendopolis. Ce cas est particulièrement pertinent car cette plateforme de crowdlending a développé un partenariat avec l'Ordre des ExpertsComptables. Ce partenariat n'ayant pas été prolongé, son étude nous a offert l'opportunité d'explorer finement d'une part les raisons de cet échec, ainsi que d'autre part d'en proposer une amélioration potentielle.

L'article montre que le partenariat entre Lendopolis et l'Ordre des expertscomptables visait deux objectifs 1) la bonne appréciation du risque de défaut des entreprises candidates à un financement, et 2) la mise en place d'un cadre permettant à l'IC de s'exprimer. Le fonctionnement théorique ou en principe de ce partenariat a reposé sur une délégation à l'EC d'un pouvoir discrétionnaire sur la sélection des projets, et sur une délégation à la foule du pouvoir de décision d'investissement dans le projet.

L'analyse des projets a révélé d'une part l'importance de l'EC dans l'expression de l'IC, et d'autre part les fonctions d'IC que celui-ci permet d'activer sous certaines conditions organisationnelles de co-dépendance. En effet, les éléments financiers et les attestations de prévisionnels fournis par les experts-comptables rendent possibles des interactions entre foule et porteurs de projet qui conduisent l'IC à remplir plusieurs fonctions bien précises. En amont de la collecte, grâce aux interactions permises par les 
outils digitaux et le rôle de l'expert, l'évaluation d'un projet par la foule a pour fonction d'améliorer la compréhension des business models des porteurs de projet, et ainsi conduire parfois à leur enrichissement, ou d'anticiper un défaut potentiel. Ainsi, la foule ne réagit pas seulement aux signaux de qualité des projets comme le montre Mollick (2014) mais également à ceux de risques. En aval de la collecte, l'IC permet une mutualisation du risque le rendant individuellement indolore pour les contributeurs lorsqu'il se concrétise (par un défaut). L'IC peut aussi prendre la forme d'un réseau d'adjuvants soutenant le porteur de projet après la collecte. Enfin, à ce stade, l'EC assure la traçabilité et le rendu de comptes.

Cependant, le partenariat a, dans les faits, été rejeté par les experts-comptables et l'exploitation de l'IC est restée par ailleurs très inaboutie. En effet, le droit de veto implicite et secret que nous avons mis en lumière s'est inscrit dans une logique low cost de délégation de la sélection des projets à une profession réglementée, sans le dire officiellement, sans la rémunérer directement et sans anticiper que cette délégation allait à l'encontre du cadre déontologique de cette profession et serait donc très mal reçue par ses membres. En outre, certaines interactions entre foule et porteurs de projet ont pu mettre en évidence des situations problématiques appelant potentiellement un arrêt de la collecte par la plateforme. Ces interactions n'ont été ni contrôlées par cette dernière ni prises en compte lorsque cela s'imposait pourtant clairement. La plateforme a donc échoué à exploiter pleinement l'IC qui s'exprimait sur les forums, contrairement aux intentions exprimées dans les discours sur le partenariat. Ainsi, la plateforme a introduit une forme d'hypocrisie organisationnelle (Brunsson, 2002), un décalage entre discours et pratiques.

Par ce double transfert à l'EC et à la foule, Lendopolis a en outre détourné le modèle de Nielsen qui implique à l'inverse un contrôle strict des porteurs de projets, pendant la sélection et pendant la campagne. Le modèle de co-dépendance s'en est trouvé déséquilibré sans que la plateforme ne mette en place de mécanisme de compensation. Ce non-respect de la co-dépendance et l'hypocrisie organisationnelle qui l'accompagne ont pu constituer des facteurs organisationnels d'échec du partenariat. A partir de cette analyse, nous proposons un modèle de co-dépendance élargie qui élimine l'hypocrisie organisationnelle tout en visant à l'expression et l'exploitation de l'IC grâce à l'EC. 


\subsection{Contributions théoriques}

Cet article contribue doublement à la littérature sur le crowdlending et à la théorie des organisations. L'article apporte tout d'abord des compléments d'explication à la déconvenue des logiques partenariales dans l'écosystème du crowdlending analysée par Calme, Onnee et Zoukoua (2018). Ces derniers justifient l'échec du partenariat de la plateforme Lendopolis avec l'Ordre des Experts Comptables par la faiblesse des externalités de réseau et de l'enrichissement du business model. En revanche, le mécanisme de veto informel et l'absence de suivi du forum par la plateforme n'ont pas été relevés par cette étude. Comme explicité ci-dessus, en découlent deux facteurs essentiels d'échec, qui s'ajoutent donc aux éléments mis en avant par Calme et al (2018) : l'hypocrisie organisationnelle (Brunsson, 2002) et le non-respect de la co-dépendance (Nielsen 2018).

La théorie des organisations permet donc de mieux comprendre les limites de la logique partenariale de Lendopolis en révélant les déviations par rapport au modèle de décisions portant sur l'appartenance, la surveillance et les sanctions notamment (Nielsen 2018). Il est intéressant de noter que le concept d'hypocrisie organisationnelle de Brunsson (2002) conserve aussi sa pertinence dans le cadre d'une organisation dite «partielle» (Ahrne et Brunsson 2010) comme le crowdlending. Nous contribuons également à revisiter le modèle d'organisation co-dépendante de Nielsen en intégrant une quatrième figure, celle de l'EC, et en proposant des modes d'organisation partielle possible entre les quatre acteurs. Ce modèle permet de repenser, sous l'angle de l'organisation, l'équilibre entre expertise et foule (Bonabeau, 2009; Malone et al., 2009).

Ainsi, l'approche par la théorie des organisations apporte des éclairages nouveaux à des études sur le crowdlending qui ont principalement mobilisé la théorie de l'agence, des plateformes ou de l'IC (Bertrand \& Jakubowski, 2016; Calme et al., 2018; Mollick, 2014; Mollick \& Nanda, 2015). En mobilisant la notion d'organisation partielle d'Ahrne et Brunsson (2010) et d'organisation co-dépendante de Nielsen (2018), nous changeons de niveau d'analyse et mettons l'accent sur les interdépendances et les mécanismes de contrôle existant entre les quatre types d'acteurs formant l'organisation co-dépendante : plateforme, porteurs de projet, foule et EC. 


\subsection{Implications pour la profession d'expert-comptable (EC)}

Dans le contexte de l'économie collaborative et de plateformes, trop peu d'études s'intéressent aux possibles évolutions de la profession d'EC. La rencontre des nouvelles technologies et de l'IC dans le financement participatif invite à repenser non seulement le contenu du métier d'EC mais aussi son utilité. Ce métier a déjà été profondément bouleversé par les nouvelles technologies, en particulier dans sa relation avec les PME et TPE (Chapellier, 2003). Il fait face à des enjeux de transformation (Ndao \& CharlesCargnello, 2015) et des menaces comme l'automatisation (Frey \& Osborne, 2017; Susskind \& Susskind, 2015). Certaines études montrent déjà des évolutions possibles vers plus de conseil aux dirigeants (Cormier et al., 2012). Cependant le développement des plateformes dans la profession, ou leur uberisation, soulève de nouveaux défis. Notre étude offre une piste d'évolution complémentaire du métier d'EC dans ce qu'il conviendrait d'appeler «l'ingénierie de l'IC», c'est-à-dire la transformation des interactions sur les plateformes en ressource et leur exploitation. Le crowdlending apparaitrait dès lors comme un avenir possible non seulement des TPE/PME pour se financer, mais aussi des experts-comptables pour se renouveler.

Cependant, ceci implique que la place des experts-comptables dans l'organisation co-dépendante soit mieux pensée et mise en valeur. Cela passe aussi par la modernisation d'un diplôme sans cesse en évolution (Degos, 2002) pour former les experts-comptables aux enjeux du digital, des nouvelles sources de financement et plus largement des nouveaux métiers de l'économie collaborative. Cet article pourra contribuer à cette modernisation du diplôme en étant par exemple discuté lors des modules de formation obligatoire des EC stagiaire. C'est un cas de réflexion stimulant pour analyser les évolutions de la profession.

Cet article montre par ailleurs que l'Ordre des experts-comptables n'a pas été en mesure de détecter et encore moins de demander l'arrêt d'un droit de veto qui n'était pas prévu dans le texte du partenariat officiellement signé mais qui a été intégré dans les faits sans que l'Ordre en soit averti. Un suivi strict des accords passés avec des tiers permettrait de prévenir des évolutions inattendues et potentiellement contraires à la déontologie de la profession. Concrètement, cela supposerait de la part de l'Ordre de formaliser des procédures de retour sur expérience en temps réel des experts-comptables ayant été sollicités dans le cadre d'un partenariat ainsi que des audits périodiques des modalités d'application des partenariats. Par ailleurs, l'échec ponctuel du partenariat sous cette forme n'implique pas nécessairement l'abandon général des logiques 
partenariales entre l'économie collaborative et la profession. Au contraire, il est possible de repenser les interactions et de remédier aux dysfonctionnements, comme nous le proposons dans cet article.

\section{Conclusion}

Cet article montre l'importance de l'expertise-comptable dans l'expression et l'exploitation de l'intelligence collective dans le crowdlending. En proposant un modèle de co-dépendance élargie qui fait place à l'expert-comptable, nous mettons l'accent non plus individuellement sur les plateformes, les porteurs de projet ou la foule, mais nous nous concentrons au contraire sur l'organisation partielle qui rassemble plateforme, porteurs de projet, foule et expert-comptable. L'interdépendance entre les quatre semble être une des conditions à l'utilisation de l'intelligence collective comme ressource dans le crowdlending. Ce modèle élargi permet aussi d'ouvrir des pistes d'évolutions pour la profession qui fait face à des défis sociétaux inévitables.

Cependant, ces résultats ne peuvent être compris sans prendre en compte les limites de l'étude, limites qui constituent autant de pistes de recherche future. Cette étude repose sur un cas unique, celui de Lendopolis dont le partenariat avec l'Ordre des Experts-Comptables a échoué. Cet échec, relu à la lumière de Nielsen (2018), nous a permis de développer une nouvelle proposition de modèle de co-dépendance. Cependant, il parait essentiel de tester théoriquement et empiriquement ce modèle, soit en faisant varier certains paramètres (temps d'interaction sur les forums, nombre d'experts-comptables, leurs positions par rapport à la plateforme) soit en l'appliquant à d'autres cas. L'importance avérée et potentielle du crowdlending et plus généralement de l'économie collaborative, mais aussi le caractère émergent de ces secteurs appellent à des approfondissements pour mieux comprendre l'articulation entre foule et expertise.

\section{Références}

Ahrne, G., \& Brunsson, N. (2010). Organization outside organizations: the significance of partial organization. Organization, 18(1), 83-104.

Ahrne, G., Brunsson, N., \& Seidl, D. (2016). Resurrecting organization by going beyond organizations. European Management Journal, 34(2), 93-101.

Akerlof, G. A. (1970). The market for "lemons": Quality uncertainty and the market mechanism. The Quarterly Journal of Economics, 488-500. 
Becker, J., Brackbill, D., \& Centola, D. (2017). Network dynamics of social influence in the wisdom of crowds. Proceedings of the National Academy of Sciences, 114(26), E5070-E5076.

Bertrand, C., \& Jakubowski, B. (2016). Le fric, c'est chic : panorama du crowdfunding en 2016. Annales des Mines - Réalités industrielles, Février 2016(1), 38-43.

Bessière, V., \& Stéphany, E. (2014). Le financement par crowdfunding. Quelles spécificités pour l'évaluation des entreprises? Revue Française de Gestion, 40(242), 149-161.

Bonabeau, E. (2009). Decisions 2.0: The power of collective intelligence. MIT Sloan Management Review, 50(2), 45.

Borins, S. (2001). Innovation, success and failure in public management research: Some methodological reflections. Public Management Review, 3(1), 3-17.

Brunsson, N. (2002). The organization of hypocrisy: Talk, Decisions and Actions in Organizations. Copenhagen Business School Press.

Calme, I., Onnee, S., \& Zoukoua, É.-A. (2018). Logiques partenariales au sein de l'écosystème du prêt entrepreneurial - Le partenariat plateformes du CLE CSOEC. Revue Française de Gestion, 44(273), 85-106. https://doi.org/10.3166/rfg.2018.00256

Chanal, V., \& Caron-Fasan, M.-L. (2010). The difficulties involved in developing business models open to innovation communities: the case of a crowdsourcing platform.M@N@Gement,13(4),318-340.

Chapellier, P. (2003). Les apports potentiels d'Internet à la mission de l'expertcomptable dans les petites entreprises. Comptabilité - Contrôle - Audit, Tome 9(2), 171-187.

Chesbrough, H. W. (2003). Open innovation: The new imperative for creating and profiting from technology. Boston: Harvard Business Press.

Cormier, D., Lapointe-Antunes, P., \& Magnan, M. (2012). Le référentiel IRS : nous dirigeons-nous vers une comptabilité au-delà du réel ? Comptabilité - Contrôle Audit, Tome 13(3), 43-55.

Cormier, D., \& Magnan, M. L. (2005). From Accounting to "Forecounting"*. Canadian Accounting Perspectives, 4(2), 243-255.

Dolder, D. van, \& Assem, M. J. van den. (2017). The wisdom of the inner crowd in three large natural experiments. Nature Human Behaviour, 1. 
Dumez, H. (2016). Méthodologie de la recherche qualitative (Vol. Seconde édition). Paris: Vuibert.

Dunleavy, P., Margetts, H., Bastow, S., \& Tinkler, J. (2006). New public management is dead-long live digital-era governance. Journal of Public Administration Research and Theory, 16(3), 467-494.

Ebondo, E., \& Pigé, B. (2002). L’arbitrage entreprise/marché: le rôle du contrôle interne, outil de réduction des coûts de transaction. Comptabilité-ContrôleAudit, 8(2), 51-67.

Eisenhardt, K. M. (1989). Building theories from case study research. Academy of Management Review, 14(4), 532-550.

Frey, C. B., \& Osborne, M. A. (2017). The future of employment: how susceptible are jobs to computerisation? Technological Forecasting and Social Change, 114, 254-280.

Galton, F. (1907). Vox populi (The wisdom of crowds). Nature, 75(7), 450-451.

Grothe-Hammer, M. (2018). Organization without actorhood: Exploring a neglected phenomenon. European Management Journal. https://doi.org/10.1016/j.emj.2018.07.009

Järvi, K., Almpanopoulou, A., \& Ritala, P. (2018). Organization of knowledge ecosystems: Prefigurative and partial forms. Research Policy, 47(8), 1523-1537. https://doi.org/10.1016/j.respol.2018.05.007

Kremer, I., Mansour, Y., \& Perry, M. (2014). Implementing the "Wisdom of the Crowd.” Journal of Political Economy, 122(5), 988-1012.

Malone, T. W., Laubacher, R., \& Dellarocas, C. (2009). Harnessing crowds: Mapping the genome of collective intelligence.

Meyssonnier, F. (2012). Le contrôle de gestion des services: Réflexion sur les fondements et l'instrumentation. Comptabilité-Contrôle-Audit, 18(2), 73-97.

Miles, M. B., Huberman, A. M., \& Saldaña, J. (2013). Qualitative data analysis: A methods sourcebook. SAGE Publications, Incorporated.

Mollick, E. (2013). Swept away by the crowd? Crowdfunding, Venture Capital and the Selection of Entrepreneurs. Working paper, the Wharton School of the University of Pennsylvania.

Mollick, E. (2014). The dynamics of crowdfunding: An exploratory study. Journal of Business Venturing, 29(1), 1-16. 
Mollick, E., \& Nanda, R. (2015). Wisdom or madness? Comparing crowds with expert evaluation in funding the arts. Management Science, 62(6), 1533-1553.

Ndao, A., \& Charles-Cargnello, E. (2015). L'Adoption Des Outils De Contrôle De Gestion Dans Les Cabinets D'Expertise Comptable. In Comptabilité, Contrôle et Audit des invisibles, de l'informel et de l'imprévisible (p. cd-rom).

Nielsen, K. R. (2018). Crowdfunding through a partial organization lens - The codependent organization. European Management Journal, OnlineFirst. https://doi.org/10.1016/j.emj.2018.01.006

Souchaud, A. (2017). Deus ex machina dans «l'espace régulatoire» du crédit en France: La reconnaissance du crowdlending face au monopole bancaire. Gérer et Comprendre. Annales Des Mines, 128, 3-13.

Surowiecki, J. (2004). The wisdom of crowds: Why the many are smarter than the few and how collective wisdom shapes business. Economies, Societies and Nations, 296.

Susskind, R., \& Susskind, D. (2015). The Future of the Professions: How Technology Will Transform the Work of Human Experts (Reprint). OUP Oxford.

Trébucq, S. (2006). Capital humain et comptabilité sociétale: le cas de l'information volontaire des entreprises françaises du SBF120. Comptabilité-Contrôle-Audit, 12(1), 103-124.

Vézina, M., \& Fortin, J. (2002). les facteurs de succès de la mise en place d'une communauté virtuelle dans les PME de services: le cas des petits et moyens cabinets d'experts-comptables. Revue Internationale PME: Économie et Gestion de La Petite et Moyenne Entreprise, 15(3-4), 63-99. 